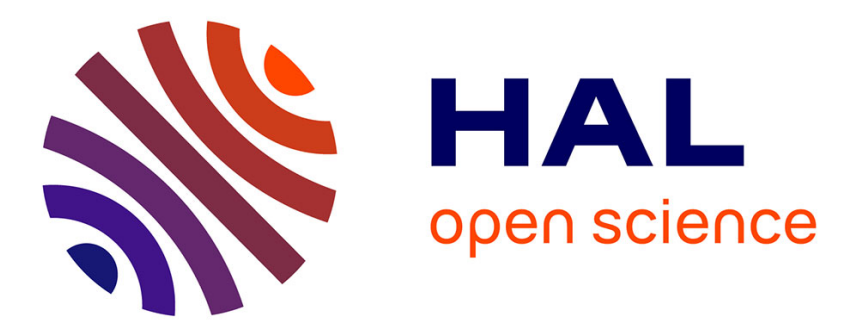

\title{
Genesis of nanogratings in silica bulk via multipulse interplay of ultrafast photo-excitation and hydrodynamics
}

Anton Rudenko, Jean-Philippe Colombier, Tatiana E Itina, Razvan Stoian

\section{To cite this version:}

Anton Rudenko, Jean-Philippe Colombier, Tatiana E Itina, Razvan Stoian. Genesis of nanogratings in silica bulk via multipulse interplay of ultrafast photo-excitation and hydrodynamics. Advanced Optical Materials, 2021, pp.2100973. 10.1002/adom.202100973 ujm-03321032

\section{HAL Id: ujm-03321032}

\section{https://hal-ujm.archives-ouvertes.fr/ujm-03321032}

Submitted on 16 Aug 2021

HAL is a multi-disciplinary open access archive for the deposit and dissemination of scientific research documents, whether they are published or not. The documents may come from teaching and research institutions in France or abroad, or from public or private research centers.
L'archive ouverte pluridisciplinaire HAL, est destinée au dépôt et à la diffusion de documents scientifiques de niveau recherche, publiés ou non, émanant des établissements d'enseignement et de recherche français ou étrangers, des laboratoires publics ou privés. 


\section{Genesis of nanogratings in silica bulk via multipulse interplay of ultrafast photo-excitation and hydrodynamics}

\section{Anton Rudenko* Jean-Philippe Colombier Tatiana E. Itina Razvan Stoian}

Dr. Anton Rudenko

Arizona Center for Mathematical Sciences and College of Optical Sciences, University of Arizona, Tucson, Arizona 85721, USA

Email: antmipt@gmail.com

Prof. Jean-Philippe Colombier, Dr. Tatiana E. Itina, Dr. Razvan Stoian

Laboratoire Hubert Curien, CNRS UMR 5516, IOGS, Université Jean Monnet 42000 St Etienne, France

Keywords: Laser materials processing, Ultrashort pulse, Fused silica, Nanostructuring, Nanogratings, Self-organization

Structuring below diffraction limit is key to developing new laser processing technologies as well as to understanding light-induced processes on mesoscopic scales, notably self-organization. Here, an advanced numerical perspective on the generation of embedded self-arranged sub-wavelength periodic patterns is developed, describing multipulse ultrafast laser interaction with bulk silica glass. Combining light and material dynamics, the approach couples self-consistently nonlinear propagation, electronic excitation, and fluid dynamics resulting in irreversible phase transitions and localized damage. With increasing the number of applied pulses, the modification changes from localized nanovoids and elongated random nanopatterns towards regular void nanogratings dominantly covering the spot of the focused laser beam. Driven by local and collective scattering events, the order imposed by electric field patterns is then amplified and stabilized by the material response. The model predicts the gradual evolution of the optical properties considering the complex interplay between material arrangement and the electromagnetic field distribution. It allows thus to define light transport optical functions optimizing losses and anisotropic effects.

\section{Introduction}

Matter redistribution under the influence of optical fields is a prominent field of research ranging from optical trapping to additive manufacturing and laser material processing. Particularly in the field of laser structuring, this process is at the origin of intriguing forms of self-organization reaching extreme sizes $[1,2]$. Topography patterns with characteristic scales well below the optical wavelength can be obtained, creating new shape and size-dependent material functions and application fields in mechanics, optics, and life sciences. The generation of regular periodic material patterns on surfaces with close-towavelength and deep sub-wavelength periodicities has been under scrutiny since more than five decades, with the most recent models advancing theories of dissipative self-organization and material rearrangement under a synergistic light action [1]. The observation of a similar phenomenon in the bulk of transparent materials, the so-called nanogratings [2], has led to the development of inclusive electromagnetic and electron plasma kinetics theories [3-5] to describe a generalized phenomenon of light-induced generation of regular patterns in two and three dimensions aligning with respect to the laser polarization $[6,7]$.

Commonly created after multipulse femtosecond laser irradiation of transparent glasses and crystals [2,3,8-13], the nanogratings emerge under a feedback action required to guide, amplify, and stabilize order. A gradual arrangement of matter under optical fields, reassembling in turn the optical patterns, matches the self-limited evolution towards complexity observed in dissipative non-equilibrium open thermodynamic systems [14]. The incipient transition to order was observed in laser-irradiated glasses by 3D-reconstruction of scanning electron microscopy and X-ray scattering images [12]. The formation of layered nanogratings has been demonstrated to generate strong birefringence [3, 15], with potential applications in high-density optical data storage [16], geometric phase optical manipulation [17, 18], and information processing [13]. Very recently, equally interesting nanovoid domains of scatterless birefringence were observed in the intermediate irradiation stages [19]. These structures are important for optical systems that optimally couple losses and anisotropy for light transport and manipulation. In view of a wide range of emerging applications, it is of interest to determine the specific drives and paths leading to the observed self-organization of matter in nonlinearly excited materials under the action of intense 
and repetitive ultrashort laser pulses. Particularly, predictive tools able to describe the properties of the patterns at different levels of their organisation are urgently needed.

Owing to the high complexity of the numerical problem, the previously reported simulations in the bulk of transparent materials were mostly limited to single pulse excitation, considered simplified damage criteria or implied reduced geometries [4-7], leaving open the questions regarding the feasibility of threedimensional multipulse arrangement with high order and regularity. A full description of a multipulse feedback mechanism should implicitly take into account not only electron plasma kinetics in spatially variable ultrafast optical fields, but also glass heating, melt flow driven by temperature gradients, culminating with local cavitation and growth of failure spots.

The present model describes the complex multiscale dynamics coupling self-consistently nonlinear propagation, electronic excitation and electron-ion thermalization, melt flow, and compressible modifications to elucidate the emergence of periodic patterns of dense and void layers in fused silica on scales below the laser wavelength. In order to investigate the nanostructure evolution upon multipulse irradition, we include both intra-pulse and inter-pulse feedbacks, considering optical transient and permanent modifications respectively. We show that the inter-pulse feedback, commonly neglected in previously proposed models, is crucial in order to achieve the regular and highly periodic nanogratings. Within the proposed model, we also validate the intermediate irradiation regimes that, via material regular structures, generate form birefringence while minimizing scattering losses.

\section{Modeling details}

The model consists of two coupled parts relevant for the excitation of electron and ion sub-systems. Thus, the first part includes nonlinear full-vector Maxwell propagation solver and electron plasma kinetics equations for momentum, energy and free carrier density. The second part considers compressible fluid dynamics described by Navier-Stokes equations with the equation-of-state for fused silica. If the electromagnetic models were already used to describe three-dimensional patterns of light in the context of periodic laser nanostructuring $[4,5]$, the thermomechanical evolution of matter driven by the temperature gradients is a recent addition that was successfully applied for laser-irradiated surfaces [20,21] and will be here validated for bulk 3D environments.

\subsection{Nonlinear propagation and energy deposition}

The numerical model is based on the three-dimensional nonlinear Maxwell equations coupled with a rate equation for the nonlinear production of free carriers for energy deposition, written as follows

$$
\left\{\begin{array}{l}
\frac{\partial \vec{E}}{\partial t}=\frac{\nabla \times \vec{H}}{\epsilon_{0} \epsilon_{\infty}}-\frac{1}{\epsilon_{0} \epsilon_{\infty}}\left(\vec{J}_{p}+\vec{J}_{p i}+\vec{J}_{K e r r}\right) \\
\frac{\partial \vec{H}}{\partial t}=-\frac{\nabla \times \vec{E}}{\mu_{0}} \\
\frac{\partial \vec{J}_{p}}{\partial t}=-\frac{\vec{J}_{p}}{\tau_{e}}+\frac{e^{2} N_{e}}{m_{e}} \vec{E} \\
\frac{\partial N_{e}}{\partial t}=w_{p i}+w_{a v}-\frac{N_{e}}{\tau_{r e c}} \\
C_{e} \frac{\partial T_{e}}{\partial t}=\nabla \cdot\left(k_{e} \nabla T_{e}\right)-\gamma_{e i}\left(T_{e}-T_{i}\right)+\vec{J}_{p} \cdot \vec{E}-E_{g} w_{a v}
\end{array}\right.
$$

where $\vec{E}$ is the electric field, $\vec{H}$ is the magnetic field, $\epsilon_{0}$ and $\mu_{0}$ are the permittivity and the permeability of free space, $\epsilon_{\infty}$ is the permittivity of the non-excited fused silica. $\vec{J}_{p}$ is the polarization current describing the electron heating by classical Drude model, $\vec{J}_{p i}$ is the photo-ionization depletion current, and $\vec{J}_{K e r r}$ introduces the Kerr effect. The expressions for the currents and the numerical method for coupling the nonlinearities with full-vector Maxwell solver can be found in Ref. [22]. The last two equations are written 
for the time-dependent electron density $N_{e}$ and temperature $T_{e} . e$ and $m_{e}$ are the electron charge and mass, $\tau_{e}$ and $\tau_{r e c}$ are the electron collision and recombination times respectively. The electrons in the conduction band are firstly generated by photo-ionization described by the Keldysh formula $w_{p i}$ [23]. The secondary electrons are produced by the avalanche ionization, when the electrons gain the critical threshold energy required for energy and momentum conservation $\approx 3 / 2 E_{g}[24-26]: \vec{J}_{p} \cdot \vec{E}=3 / 2 E_{g} w_{a v}$, where $E_{g}$ is fused silica electron bandgap. Dealing with the electronic excitation well below the complete ionization of fused silica, we omit the negligible pre-factor $\frac{N_{v}-N_{e}}{N_{v}}\left(N_{v}\right.$ being the electron density in the valence band) for ionization rates, losses, valence band permittivity, and Kerr response. In Equation (1), we use a simplistic single rate equation for carrier excitation limited by extreme computational memory and time requirements for three-dimensional simulations. More accurate quantitative description of energy deposition and ionization processes can be achieved by considering multiple rate equations [25, 26] and energy-resolved Bloch-Boltzmann approaches [24,27], but occurs to be outside of the scope of the current discussion.

The equation for the electron temperature includes electron diffusion, electron-ion coupling term, Joule heating and losses due to avalanche ionization. The electron heat capacity is approximated by $C_{e}=3 k_{B} n_{e} / 2$, where $k_{B}$ is the Boltzmann constant. The electron heat conductivity is $k_{e}=2 k_{B}^{2} \mu_{e} n_{e} T_{e} / e$, where $\mu_{e}$ is the electron mobility. The electron-ion coupling factor is related to the electron capacity as $\gamma_{e i}=C_{e} / \tau_{e i}$. The parameters for fused silica are used as in Ref. [28].

\subsection{Hydrodynamic equations}

A major development of the code with respect to the electromagnetic description is the accountability of fluid dynamics permitting the spatial evolution of laser-induced structures. To take into account the melt flow in fused silica heat-affected regions, compressible Navier-Stokes equations are solved, written in the form of the conservation laws for energy, momentum and mass as follows

$$
\left\{\begin{array}{l}
\rho C_{i}\left[\frac{\partial T_{i}}{\partial t}+\vec{u} \cdot \nabla T_{i}\right]=\nabla \cdot\left(k_{i} \nabla T_{i}\right)+\gamma_{e i}\left(T_{e}-T_{i}\right) \\
\frac{\partial(\rho \vec{u})}{\partial t}+(\vec{u} \cdot \nabla)(\rho \vec{u})+(\rho \vec{u}) \nabla \cdot \vec{u}= \\
=-\nabla P_{i}+\mu \nabla^{2} \vec{u}+\frac{1}{3} \mu \nabla(\nabla \cdot \vec{u}) \\
\frac{\partial \rho}{\partial t}+\nabla \cdot(\rho \vec{u})=0,
\end{array}\right.
$$

where $\vec{u}$ is the fluid velocity, $\rho, T_{i}$ and $P_{i}$ are the density, temperature and pressure of fused silica respectively. The dependence $P_{i}\left(\rho, T_{i}\right)$ is evaluated from the equation-of-state for fused silica [29]. $\mu\left(T_{i}\right)$ is the fused silica viscosity, which decays exponentially with the increasing temperature and here is evaluated according to Ref. [30]. For the reference, the viscosity is as high as $\mu \approx 10^{8} \mathrm{~Pa} \cdot \mathrm{s}$ near the softening point $T_{\text {melt }}=1875 \mathrm{~K}$ and decreases down to $\approx 10^{3} \mathrm{~Pa} \cdot \mathrm{s}$ at the highest relevant temperatures of $T_{i} \approx 3000 \mathrm{~K}$. Additionally, heat capacity $C_{i}\left(T_{i}\right)$ and thermal conductivity $k_{i}\left(T_{i}\right)$ dependencies are taken into account, based on Refs. [31,32].

\subsection{Glass decomposition criteria}

After each pulse, irreversible modifications occur in the bulk of silica, resulting in the appearance of void structures. The next pulse is considered to interact with newly arranged inhomogeneous distribution of void-like structures with the permittivity of air/gas $\epsilon=1$. We imply two criteria to define the established rarefaction regions: one for the appearance of random nucleation scattering centers or nanopores and the other one is for localized cavitation processes in liquid phase discussed in details in Ref. [33].

The first criterion is required for the initial perturbation of the system, where the probability is related to the temperature as in the classical nucleation theory $\propto \exp \left(k_{B} T_{i} / W\right)$, where $W=4 \pi \gamma r^{2} / 3$ is the free energy of the critical nucleus of $r=20 \mathrm{~nm}$ (equal to the mesh cell size) and $\gamma$ is the surface tension. 
The minimum probability is set to be $10^{-4}$ for liquid fused silica at $T_{\text {melt }}=1875 \mathrm{~K}$. We consider the temperature dependence of surface tension $\gamma=\gamma_{0}\left(1-T_{i} / T_{c r}\right)^{1.25}$ with $\gamma_{0}=0.3 \mathrm{~N} / \mathrm{m}$ and the critical temperature $T_{c r}=5400 \mathrm{~K}$ for fused silica.
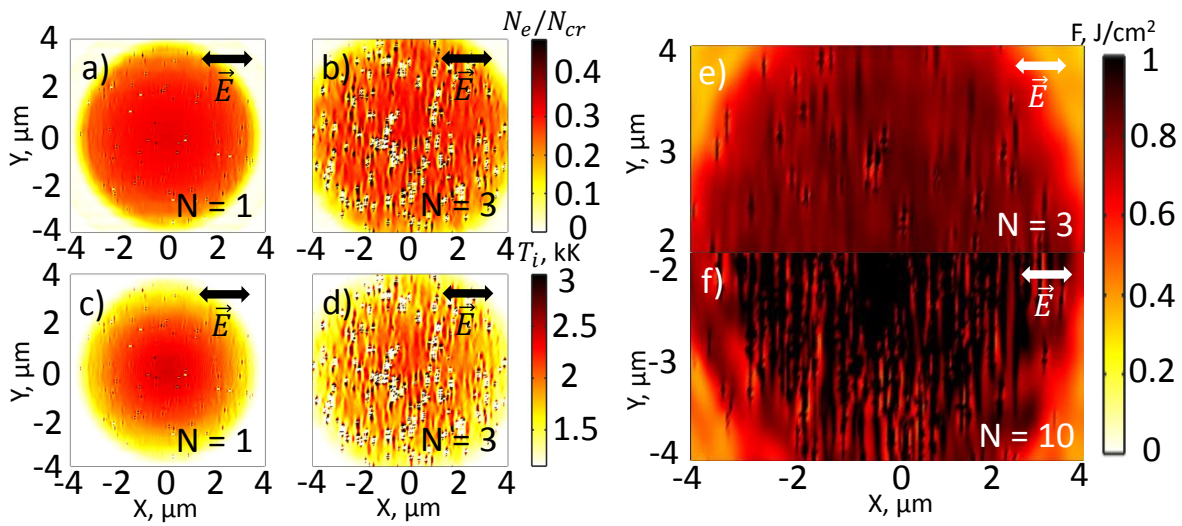

Figure 1: (a-d) Electron density and temperature snapshots of the irradiated regions subject to one pulse $N=1$ and few pulses $N=3$ irradiation. The maximum values are plotted. The electron density is normalized to the critical value $N_{c r}$. (e-f) Energy deposition snapshots (in terms of fluence) showing the effect of the number of applied pulses on the ordering of periodic nanoplasmas in the chosen half-Gaussian spots. The pulse energy is $250 \mathrm{~nJ}$, the pulse duration is $200 \mathrm{fs}$. The number of pulses $N$ is indicated in each image.

The second criterion for cavitation is the Grady's criterion for spall in Newtonian liquids derived from the viscoelastic energy conservation law [34]. Such criteria have been routinely applied for sub-surface cavitation in metals, using the critical negative pressures and the strain rates within material as the indicators of the mechanical failure $[21,35]$. Following similar approach, the critical negative pressure for surface-energy spall is $|P| \geq\left(6 \rho^{2} c_{s}^{3} \gamma \zeta\right)^{1 / 3}$ and for viscous-dissipation spall is $|P| \geq\left(2 \rho c_{s}^{2} \mu \zeta\right)^{1 / 2}$, where $c_{s} \approx 5.9 \cdot 10^{3} \mathrm{~m} / \mathrm{s}$ is the longitudinal speed of sound in fused silica and $\zeta=\delta \rho / \rho \delta t$ is the strain rate due to rarefaction $\delta \rho$ during the exposure time $\delta t$.

These two criteria are applied to every point of bulk silica as soon as the conditions are met and the structure topography changes according to these criteria at the beginning of the next pulse. For simplicity, we do not consider the possibility of void replenishment by denser material or its movement in melt flow as soon as the void structure is organized by previous pulses. Modeling of these processes would require knowledge of precise intrinsic material properties/multi-component chemistry in the damage regions and significantly more refined mesh. Note that the involvement of chemical processes including $S i-O$ bonds decomposition was reported and supported by the observations of the molecular oxygen content in the void-like structures $[10,36]$. To a first approximation, the void content is considered here as the material with $\epsilon \approx 1$, negligibly low electronic and material density (compared to fused silica). Additionally, the increase of the refractive indices in the densified regions is not taken into account but is discussed in the next sub-section.

\section{Results and Discussion}

The formation of quasi-periodic nanoplasmas with periodicities $\lambda / 2 n$, where $\lambda$ is the laser wavelength and $n$ is the refractive index of the material, starting from initial inhomogeneous refractive index distributions (related to rough interface [6,7], presence of intrinsic voids or defects $[4,5]$, or refractive index perturbations [37]) was reported in several works. Independently on the nature of the initial perturbations, the interference of the incident and the inhomogeneity-scattered waves prioritizes the arrangement with periods being a fraction of the propagating laser wavelength in medium. Ordering occurs upon nanoplasma (intra-pulse feedback) or nanovoid structure (inter-pulse feedback) growth perpendicular to the laser polarization, both enhancing the scattering effects. In this work, these feedback mechanisms supported by cumulative generation and evolution of structures driven by light are combined together via multipulse simulations indicating stronger convergence to regular patterns. 


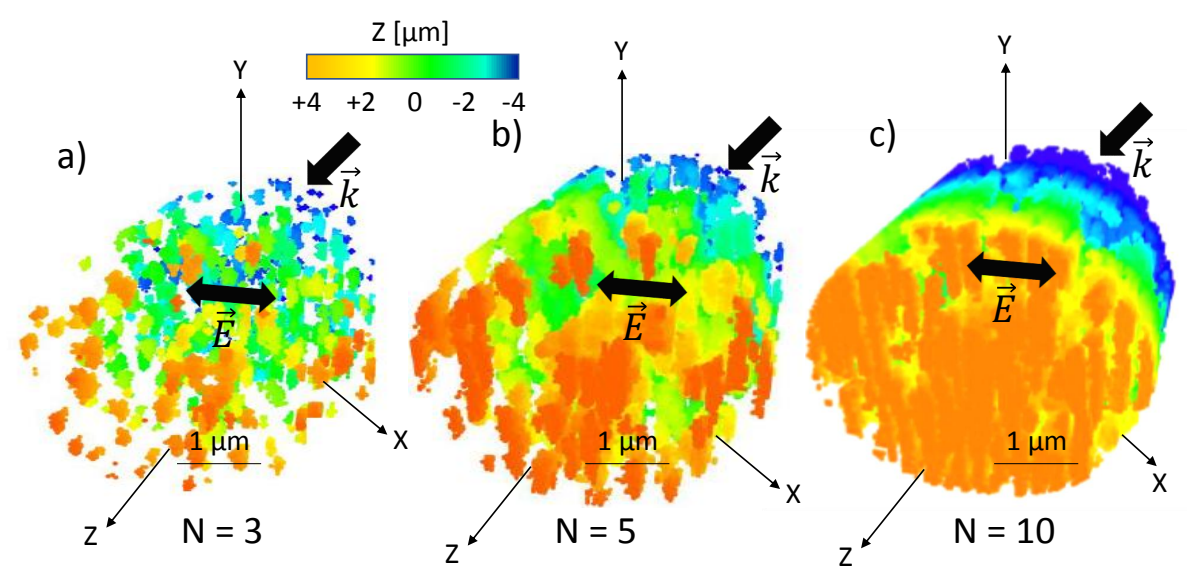

Figure 2: Three-dimensional void structures $(\epsilon=1)$ (a) at lower number of applied pulses (individual nanopores); (b) intermediate number (ellipsoidal nanoplanes); (c) high number (nanogratings). The colors are applied along the propagation direction $\vec{k}$ for better visibility. The direction of laser polarization $\vec{E}$ and the number of pulses $N$ are indicated in each image.

The proposed scenario is motivated by the observations of laser-induced hollow nanocavities and nanopores as integral parts in the nanogratings genesis $[9,10]$. These results suggest already an intrication of near-field effects (hence eventual periodicities below the half wavelength in medium) and material fracture effects (void scales in the $10 \mathrm{~nm}$-range) [3]. The present model sustains a perspective where ultrashort laser interacts with distributed intrinsic and laser-induced scattering centers. Polarization-dependent local field enhancement near the individual nanovoids contributes to the optical anisotropy of the system. More particularly, the orientation of the enhanced field occurs to be perpendicular to the laser polarization as a consequence of the optical response of a small inhomogeneity with a lower refractive index (void structure) inside transparent medium with higher refractive index (non-excited glass). Local field enhancement near the individual inhomogeneities results in enhanced nonlinear absorption and localized optical breakdown. The ionization process locally transforms the dielectric material into an absorbing plasma with quasi-metallic properties. The electrons in the conduction band are heated by the laser field and transfer their energy to the matrix. As a result of rarefaction processes driven by negative pressures, the localized nanovoids are extended in the direction of higher thermal gradients. Upon the next pulse, ultrashort light interacts with the modified nanostructures, resulting in partial symmetry breaking in the silica bulk. The repetitive interaction contributes to the growth of nanoplanes, scattering efficiently light in the direction perpendicular to laser polarization. The interference of the incident light with the waves scattered by these elongated nanostructures results in a standing light pattern inducing enhanced nonlinear absorption in quasi-periodic nanoplanes perpendicular to laser polarization. After each pulse, the established temperature and pressure gradients contribute to material rearrangements that assist in creating a higher level of complexity and order in the incoming light redistribution, determining a gradual evolution to order.

The laser irradiation conditions in silica bulk are chosen to be below the ablation threshold and microexplosion regimes in a pure sample, with a pulse energy of $250 \mathrm{~nJ}, 200 \mathrm{fs}$ pulse duration and moderate focusing $N A \approx 0.65$ of a Gaussian beam. Starting with few nucleation scattering centers, Fig. 1(a,c) shows the maximum free carrier density and lattice temperature distributions upon single-pulse irradiation. The typical densities are sub-critical $\left(N_{c r} \approx 1.74 \cdot 10^{27} \mathrm{~m}^{-3}\right.$ being the critical density in vacuum for the respective laser wavelength); upon multipulse irradiation only localized hot spots reach higher values. The estimated maximal temperatures lie in the $2000-2500 \mathrm{~K}$ interval enabling low viscosity states. For comparison, the snapshots of electron density and temperatures are also shown for the case of higher number of applied pulses just before the onset of the periodic structures in Fig. 1(b,d). Once formed through local cavitation, the nanovoids are considered as non-ionizing medium and, therefore, they appear as points with reduced densities and temperatures. The periodic arrangement of the electronic modification starts with the number of pulses smaller than required for the establishment of continuous permanently inscribed nanovoids. In fact, the nanoplasmas affect the energy deposition with already established asymmetrical response as shown in Fig. 1(e). Nevertheless, Fig. 1(f) shows that much higher regularity and contrast 
can be achieved within several pulses with the considered inter-pulse feedback.

Three-dimensional void morphologies created in bulk silica, corresponding to different number of applied pulses, are illustrated in Fig. 2. At each pulse, light interacts with these complex structures that evolve upon multipulse laser irradiation. First pulses would encounter random isolated structures, slightly extended in the direction of laser propagation. They evolve into the oriented nanoplanes in Fig. 2(b) and finally, form continuous well-ordered gratings in Fig. 2(c). Because of high complexity, the two-dimensional snapshots in the plane perpendicular to laser propagation are further discussed in details.
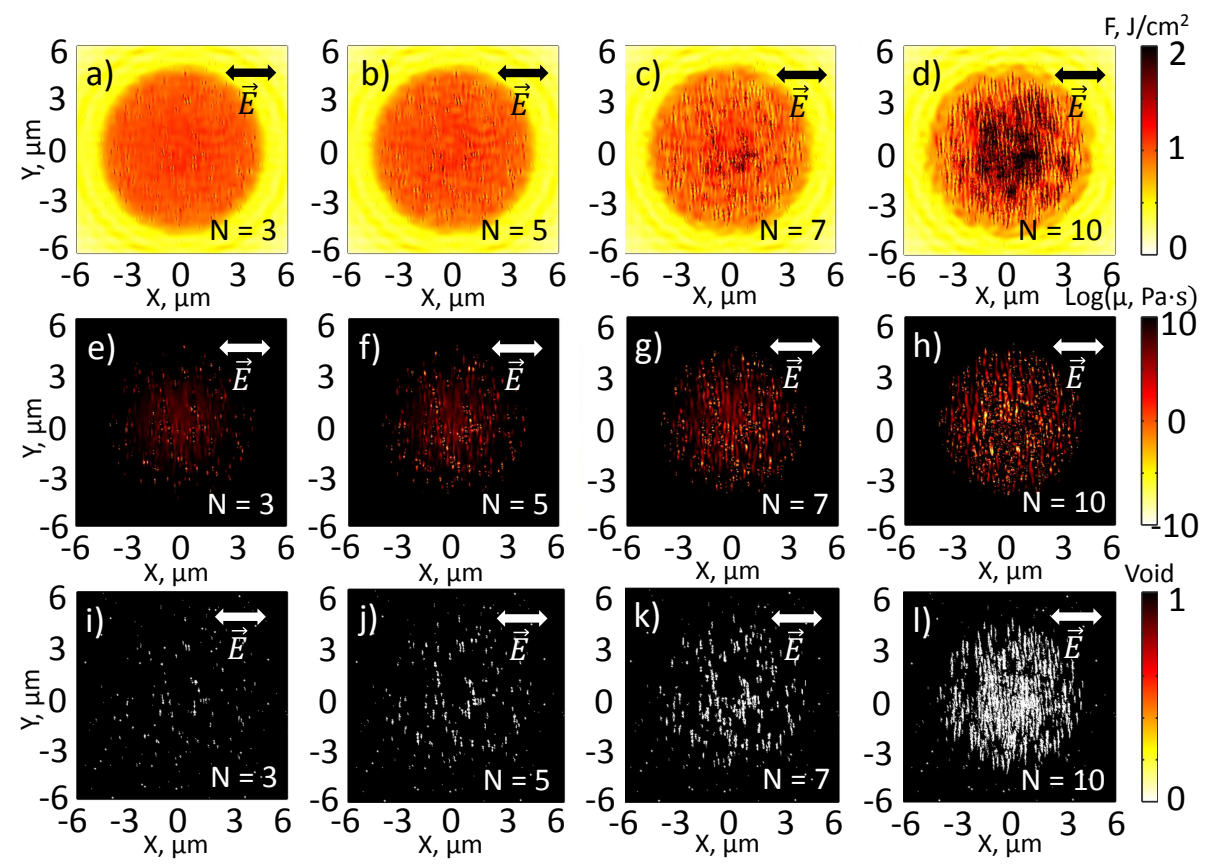

Figure 3: Multipulse snapshots corresponding to (a-d) the deposited energy density during the laser pulse excitation inside the bulk; (e-h) viscosity maps underlying the material under transitions to the liquid phase after pulse excitation; (i-l) final structure where 1 stands for undamaged material and 0 for a void structure. The pulse energy is $250 \mathrm{~nJ}$, the pulse duration is $200 \mathrm{fs}$. The number of pulses $N$ is indicated in each image.

The results of multipulse simulations with the increasing number of applied pulses from left to right are summarized in Fig. 3. The upper part of the figure shows how light interacts with inhomogeneous silica bulk during the ultrashort pulse excitation in terms of fluence. The central part stands for viscosity of the melt flow which underlines the regions affected by softening and transitions to the liquid phase. Finally, the bottom part of the figure highlights the final void structure established due to irreversible damage. During first pulses (for instance, Fig. 3(a,e,i)) the interaction is limited to localized hot spots randomly distributed along almost homogeneous Gaussian section. These hot spots originate from local field enhancement near nanoscopic nucleation centers and lead to local cavitation and growth of nanovoid structures perpendicular to laser polarization. As a result of such a feedback, the ellipsoidal nanovoids are pre-formed in Fig. 3(j,k). These structures interact much stronger with the incident light, and the interference effects result in well-defined profiles of periodic energy deposition in Fig. 3(c). The region with melt flow expands and the thermal gradients are pronounced on the viscosity maps in Fig. 3(g), indicating that the hotter regions are separated by the regions with extremely high viscosity in almost solid state. The gradients originate from contrasted nanoplasmas that absorb efficiently light but are surrounded by nearly transparent silica bulk. This feature is different from the mechanisms predicted numerically on the laser-irradiated metal surfaces [21]. In case of metals, the viscous dissipation is much weaker due to lower viscosity and its weaker dependency on the temperature. As a result, the melt flow is actively driven by pressure gradients and Marangoni forces through the laser-affected regions. In glass, the melt flow is confined between solid regions, and the relaxation occurs mostly mechanically, through the dissipation of pressure waves in the surrounded regions. The emergence of the continuous void structures in Fig. 3(l) requires few additional pulses, during which ones the structures become regular, adapting their periods to a 
fraction of the laser wavelength in medium. Interestingly, when fully established, the structures resonantly enhance scattering and absorption as seen in Fig. 3(d). Larger number of pulses is expected to melt larger areas, including in between the structures, and create additional damage. Melting the whole domain above temperatures $\approx 2500 \mathrm{~K}$ might result in the erasure of self-organized nanogratings [33].
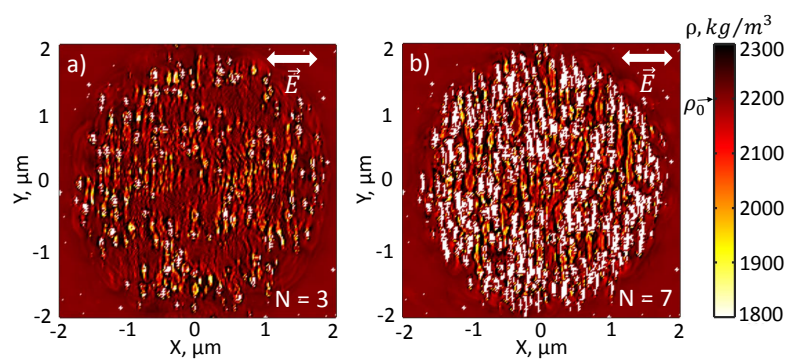

Figure 4: Silica density snapshots corresponding to $50 \mathrm{ps}$ after irradiation by (a) $N=3$ and (b) $N=7$ pulses. The pulse energy is $250 \mathrm{~nJ}$, the pulse duration is $200 \mathrm{fs}$. The density of pristine silica $\rho_{0} \approx 2200 \mathrm{~kg} / \mathrm{m}^{3}$ is indicated on the scale.

Fig. 4 shows the magnified silica density snapshots taken 50 ps after ultrashort pulse excitation by (a) $N=3$ and (b) $N=7$ pulses (the time delay is given after the last pulse exposure). Here, the white colored regions are voids; therefore, the first snapshot corresponds to the situation where only localized random voids appear; the pre-formed perpendicularly oriented structures are already established in the second one. Apart from these patterns, the transient densities below and above the pristine silica density are clearly seen. The first ones correspond to the electronic plasma patterns established during laser pulse excitation and heated above the softening temperature, subject to cavitation if significantly negative pressures are reached. The second ones are transiently densified regions between these plasmas, regions where the compressive stresses are accumulated due to shock waves propagating from the plasmas. This accumulation might result in the permanent plastic deformations and positive refractive index changes [29,38]. The index contrast of order $10^{-4}-10^{-3}$ was predicted by thermo-elasto-plastic simulations for the regions with tensile stress exceeding the critical yield strength [39].

The timescales of nanopattern relaxation and void formation by cavitation are detailed by dynamic snapshots of the negative pressures $P_{i}\left(\rho, T_{i}\right)$ inside silica bulk in Fig. 5 . The response is strongly inhomogeneous following the complex temperature distribution. For instance, first voids appear from the hot spots immediately after the electron-ion thermalization $\approx 15$ ps in Fig. 5(a). However, the larger elongated structures with a pronounced polarization-dependent response necessitate longer timescales, in between 30 ps and 50 ps as underlined in Fig. 5(b,c). The critical negative pressures required for cavitation also vary dependent on the attained temperatures and effective relaxation time from $-3 \mathrm{GPa}$ to $-100 \mathrm{MPa}$.
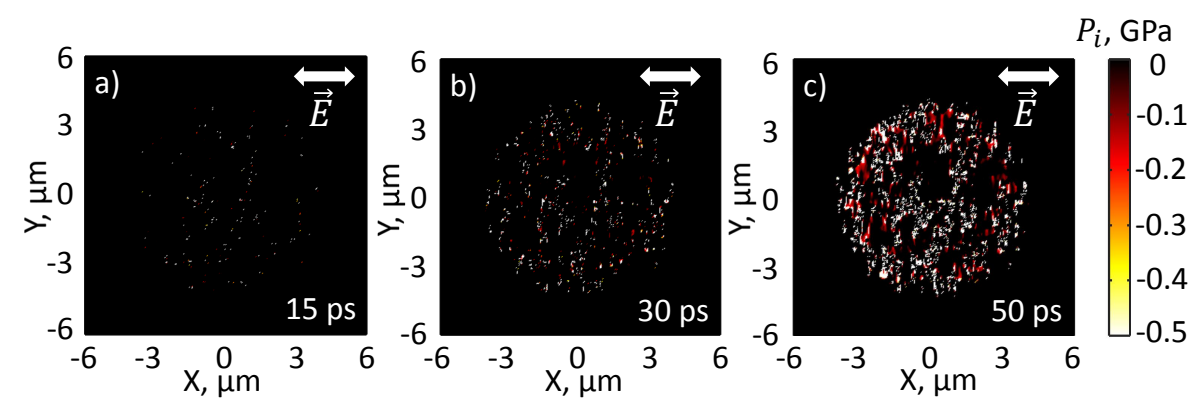

Figure 5: Snapshots of negative pressures as precursors of laser-induced cavitation inside bulk silica at the times (a) 15 ps; (b) $30 \mathrm{ps}$, and (c) $50 \mathrm{ps}$ after excitation by $N=5$ pulse. The pulse energy is $250 \mathrm{~nJ}$, the pulse duration is $200 \mathrm{fs}$.

In addition to void formation and elasto-plastic effects, an alternative pathway for positive density changes in fused silica is caused by electronic excitation and bond breaking $[36,40]$. These are a consequence of electron self-trapping [41] and occur dominantly in the solid phase. Their level corresponds to a gentle process of densification in the range of $10^{-4}$ per pulse and its role is minor in the present discussion compared to the strong changes occurring via cavitation and melt flow. The process occurs at relatively 
low carrier densities that keep the solid cold, where exciton self-trapping is accompanied by a stabilizing atomic displacement and structural distortion, locally densifying the material. It is to be noted that, with stronger excitation, fast vibrational activation will decrease the yield of structural densification by lowering the probability of carrier-self-trapping (with a complete arrest in the softening phase), limiting thus the index contrast achievable by this path $[42,43]$. Thermomechanical effects will then kick-in. The cooling cycle may equally determine soft densifications via a change of the fictive temperature providing the process is sufficiently long. Adding to the electronically assisted structural densification, for a range of temperatures approaching the softening value with enough molecular mobility in the low viscosity glass, rapid cooling may induce a structurally denser phase [44], particularly for the anomalous thermal behavior of fused silica. The quantitative estimation of such refractive index changes is not discussed here and should rely on the realistic estimation of the number of the required pulses or on long-time processes of lattice cooling and relaxation freezing new structural arrangements. Small refractive index perturbations $\ll 0.01$ do not have a strong effect on nonlinear pulse propagation at the nanoscale, whereas scattering originates mostly from void-like domains suggesting thus the dominance of the thermomechanical scenario.

Given the importance of thermally driven material changes, potential regimes of thermal accumulation may be of interest. Thus, the question of laser repetition rate becomes relevant for multipulse irradiation. The experimental observations conclude that melting enhanced by heat accumulation during high repetition rate processing prevents nanogratings formation or erases the created structures [45]. Importantly, the formation of nanogratings is commonly observed for low repetition rates $(<200 \mathrm{kHz}$, when heat accumulation can be safely avoided), and our simulations are performed neglecting heat accumulation effects to emphasize the pure role of interpulse feedback solely driven by intrinsic topography and optical refractive index modifications. The potential contribution of heat accumulation to nanogratings erasure in terms of the attained temperature threshold has been discussed in a recent work [33]. Simulations in the current work (see Fig. 3(1)) indicate that high temperatures (that are reachable as well by high repetition rate processing) would produce larger void-like modifications with sizes comparable to the spacing $\approx 250$ $\mathrm{nm}$ between the created nanostructures, resulting in merging with separate structures to form a single micrometer disrupted region.
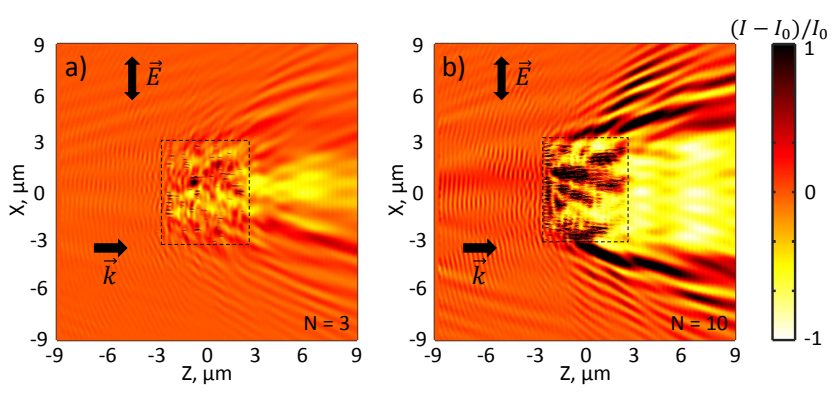

Figure 6: Scattering losses upon propagation of plane continuous waves through the nanovoid structures created (a) by $N=3$ and (b) $N=10$ pulse irradiations. The intensity is averaged over the period and normalized to the incident value $I_{0}$. The regions limited by dashed lines indicate the nanostructured domains.

The generation of nanostructures was associated with optical functions emerging from phase and polarization sensitivity [46]. The recent report [19] of low-loss birefringence raises the interest in the intermediate stages in the nanogratings formation. We have tested qualitatively the optical properties of the nanovoid structures created at different stages of their multipulse evolution by simulating the passage of a continuous wave beam (plane wave) with a particular orientation of the polarization vector with respect to the grating vector. As expected, the structures exhibit strong birefringence during their genesis towards regular nanostructured domains. The higher the number of pulses is, the more compact structures become as shown in Fig. 2. The numerical results for plane wave propagation through the void structures detailed in Fig. 6 confirm that the scattering losses increase as well with the increasing number of pulses. This dependency is in agreement with the experimental observations in Ref. [19].

Two last points should be finally addressed.

The experiments report the formation of nanogratings after higher number of applied pulses (thou- 
sands) $[2,3,12,45]$ than in the current simulations. This discrepancy implies that the inter-pulse feedback should be much weaker. One possible reason is that a higher number of pulses is required to promote the appearance of initial scattering centers (inhomogeneities). This might be related also to a much more delicate and complex intrinsic evolution from initial defects to nanoscale damage inside transparent materials [8]. Although this point is not yet well understood in the literature and the inter-pulse feedback in the current work is likely overestimated in order to make a problem still computationally feasible, we provide an essential improvement comparing to single pulse models $[4,5,7]$, where no discussion regarding the involved hydrodynamics and the potential impact on the next pulse evolution was given. It is worth noting that much slower evolution (hundreds of pulses instead of 10 pulses) towards well-organized nanostructures can be achieved even within the same numerical model, by considering much refined spatial resolution (sub-nm instead of $20 \mathrm{~nm}$ ) and, consequently, the nucleation centers of few nanometer size that are produced by heterogenous nucleation. This would result in a weaker scattering but still does not exclude the pronounced role of local field surface effects on a few-nanometer scale. In this case, the growth rate of nanoplasmas and void nanostructures is expected to be slower, resulting in higher number of pulses incubation effects and much thinner nanogratings, similar to the ones observed experimentally [3]. This way, we believe that the better quantitative description in terms of the number of applied pulses is rather a technical detail than the missing physical mechanism.

The nanograting period reduction with the increasing number of applied pulses reported in Refs. $[2,45]$ is another intriguing dependency yet to be clarified. For instance, the nanoplasmas with periods smaller than $\lambda / 2 n$ were reported to originate in systems with a higher concentration of inhomogeneities, enhanced by multiple scattering [5]. Intuitively, a mechanism similar to the one reported on laser-irradiated metal surfaces with a blueshift caused by collective response of random nanoholes and consequent period reduction [47] can be applicable to describe the observed dependency. However, the smooth successive transition from already created regular void nanostructures with larger periods towards the structures with lower periods is challenging to describe inside bulk material without consideration of possible void replenishment by denser material. Thus, an additional inter-pulse feedback based on readjustment of irregular void structure interfaces by melt flow driven by surface tension effects [21] together with irreversible damage effects considered in the current work might be relevant to further improve the proposed model for fused silica.

\section{Conclusion}

In conclusion we have performed multipulse simulations to describe the interplay between ultrafast light and matter in the formation of light-driven self-organized nanostructures in bulk silica glass. Uniquely mixing light and matter dynamics, the model couples full-vector nonlinear propagation in inhomogeneous medium, electronic excitation by ionization processes, energy transfer to lattice, and fluid dynamics. Each laser pulse results in the appearance of new regions exposed to irreversible localized damage and void formation. New arrangement generates inhomogeneous field distributions, affecting the electron plasma kinetics and fluid dynamics after electron-phonon thermalization. In this scenario, the anisotropy is set by the electromagnetic field and the degree of order facilitated by material topography evolution. Light acts as both the generator and the stabilizer of the structures. Our simulations indicate that better order and anisotropic refractive index contrast are achieved after multipulse irradiation, during which the modification passes through the stages of disorder with random nanovoid distribution, polarization-dependent deformations resulting in the formation of elongated ellipsoidal voids, and regulation into connected nanogratings with deeply sub-wavelength periodicity. The model facilitates predictions for the laser-induced formation of optical elements optimizing losses and vectorial properties.

\section{Acknowledgements}

This work was supported by an Air Force Office of Scientific Research (AFOSR) award FA9550-19-10032 and by ADEME: Agence de la transition écologique via the project IMOTEP. This work has equally been funded by a public grant from the French National Research Agency (ANR) under the Investments for the Future Program (PIA), which has the reference EUR MANUTECH SLEIGHT - ANR-17-EURE-0026. 
We also acknowledge the support of LABEX MANUTECH-SISE (ANR-10-LABX-0075) of the Université de Lyon, within the program "Investissements d'Avenir" (ANR-11-IDEX-0007).

Conflict of interest

The authors declare no conflict of interest.

\section{References}

[1] J. Bonse, S. Gräf, Laser EG Photonics Reviews 2020, 14, 102000215.

[2] Y. Shimotsuma, P. G. Kazansky, J. Qiu, K. Hirao, Physical Review Letters 2003, 91, 24247405.

[3] R. Taylor, C. Hnatovsky, E. Simova, Laser $\&$ Photonics Reviews 2008, 2, 1-2 26.

[4] R. Buschlinger, S. Nolte, U. Peschel, Physical Review B 2014, 89, 18184306.

[5] A. Rudenko, J.-P. Colombier, T. E. Itina, Physical Review B 2016, 93, 7075427.

[6] A. Rudenko, J.-P. Colombier, S. Höhm, A. Rosenfeld, J. Krüger, J. Bonse, T. E. Itina, Scientific Reports 2017, 7, 11.

[7] J.-L. Déziel, L. J. Dubé, S. H. Messaddeq, Y. Messaddeq, C. Varin, Physical Review B 2018, 97, 20 205116.

[8] V. R. Bhardwaj, E. Simova, P. P. Rajeev, C. Hnatovsky, R. S. Taylor, D. M. Rayner, P. B. Corkum, Physical Review Letters 2006, 96, 5057404.

[9] S. Richter, A. Plech, M. Steinert, M. Heinrich, S. Döring, F. Zimmermann, U. Peschel, E. B. Kley, A. Tünnermann, S. Nolte, Laser $\&$ Photonics Reviews 2012, 6, 6787.

[10] M. Lancry, B. Poumellec, J. Canning, K. Cook, J.-C. Poulin, F. Brisset, Laser \& Photonics Reviews 2013, 7, 6953.

[11] M. Lancry, F. Zimmerman, R. Desmarchelier, J. Tian, F. Brisset, S. Nolte, B. Poumellec, Applied Physics B 2016, 122, 31.

[12] F. Zimmermann, A. Plech, S. Richter, A. Tünnermann, S. Nolte, Laser \& Photonics Reviews 2016, 10, 2327.

[13] B. Zhang, D. Tan, X. Liu, L. Tong, P. G. Kazansky, J. Qiu, Advanced Optical Materials 2019, 7, 20 1900593.

[14] I. Prigogine, Science 1978, 201, 4358777.

[15] M. Beresna, M. Gecevičius, M. Lancry, B. Poumellec, P. G. Kazansky, Applied Physics Letters 2013, 103, 13131903.

[16] J. Zhang, M. Gecevičius, M. Beresna, P. G. Kazansky, Physical Review Letters 2014, 112, 3033901.

[17] F. Zhang, A. Cerkauskaite, R. Drevinskas, P. G. Kazansky, J. Qiu, Advanced Optical Materials 2017, 5, 231700342.

[18] R. Drevinskas, M. Beresna, J. Zhang, A. G. Kazanskii, P. G. Kazansky, Advanced Optical Materials 2017, 5, 11600575.

[19] M. Sakakura, Y. Lei, L. Wang, Y.-H. Yu, P. G. Kazansky, Light: Science \& Applications 2020, 9, 1 1.

[20] A. Rudenko, C. Mauclair, F. Garrelie, R. Stoian, J.-P. Colombier, Physical Review B 2019, 99, 23 235412. 
[21] A. Rudenko, A. Abou-Saleh, F. Pigeon, C. Mauclair, F. Garrelie, R. Stoian, J.-P. Colombier, Acta Materialia 2020, 19493.

[22] A. Rudenko, J.-P. Colombier, T. E. Itina, International Journal of Numerical Modelling: Electronic Networks, Devices and Fields 2018, 31, 2 e2215.

[23] L. V. Keldysh, Soviet Physics JETP 1965, 201307.

[24] A. Kaiser, B. Rethfeld, M. Vicanek, G. Simon, Physical Review B 2000, 61, 1711437.

[25] B. Rethfeld, Phys. Rev. Lett. 2004, 92 187401.

[26] B. H. Christensen, P. Balling, Physical Review B 2009, 79, 15155424.

[27] E. Smetanina, P. G. de Alaiza Martínez, I. Thiele, B. Chimier, A. Bourgeade, G. Duchateau, Physical Review E 2020, 101, 6063206.

[28] A. Rudenko, H. Ma, V. P. Veiko, J.-P. Colombier, T. E. Itina, Applied Physics A 2018, 124, 163.

[29] R. Beuton, B. Chimier, J. Breil, D. Hébert, P.-H. Maire, G. Duchateau, Journal of Applied Physics 2017, 122, 20203104.

[30] M. I. Ojovan, W. E. Lee, Journal of applied physics 2004, 95, 73803.

[31] J. Zhao, J. Sullivan, J. Zayac, T. D. Bennett, Journal of Applied Physics 2004, 95, 105475.

[32] P. Combis, P. Cormont, L. Gallais, D. Hebert, L. Robin, J.-L. Rullier, Applied Physics Letters 2012 , 101, 21211908.

[33] A. Rudenko, J.-P. Colombier, T. E. Itina, Physical Chemistry Chemical Physics 2018, $20,85887$.

[34] D. Grady, Journal of the Mechanics and Physics of Solids 1988, 36, 3353.

[35] M. E. Povarnitsyn, T. E. Itina, M. Sentis, K. V. Khishchenko, P. R. Levashov, Physical Review B 2007, 75, 23235414.

[36] K. Mishchik, C. D’Amico, P. K. Velpula, C. Mauclair, A. Boukenter, Y. Ouerdane, R. Stoian, Journal of Applied Physics 2013, 114, 13133502.

[37] V. Gildenburg, I. Pavlichenko, Optics Letters 2019, 44, 102534.

[38] N. M. Bulgakova, V. P. Zhukov, S. V. Sonina, Y. P. Meshcheryakov, Journal of Applied Physics 2015, 118, 23233108.

[39] S. Najafi, A. S. Arabanian, R. Massudi, Journal of Physics D: Applied Physics 2016, $49,25255101$.

[40] J. W. Chan, T. R. Huser, S. H. Risbud, D. M. Krol, Applied Physics A 2003, 76, 3367.

[41] P. Audebert, P. Daguzan, A. Dos Santos, J. C. Gauthier, J. P. Geindre, S. Guizard, G. Hamoniaux, K. Krastev, P. Martin, G. Petite, A. Antonetti, Physical Review Letters 1994, 731990.

[42] P. Jürgens, M. Vrakking, A. Husakou, R. Stoian, A. Mermillod-Blondin, Applied Physics Letters 2019, 115, 19191903.

[43] P. K. Velpula, M. K. Bhuyan, F. Courvoisier, H. Zhang, J. P. Colombier, R. Stoian, Laser E Photonics Reviews 2016, 10, 2230.

[44] R. Brückner, Journal of Non-Crystalline Solids 1971, 5, 3177.

[45] S. Richter, M. Heinrich, S. Döring, A. Tünnermann, S. Nolte, U. Peschel, Journal of Laser Applications 2012, 24, 4042008.

[46] T. Gerke, R. Piestun, Nature Photonics 2010, 4, 20188.

[47] A. Rudenko, C. Mauclair, F. Garrelie, R. Stoian, J.-P. Colombier, Nanophotonics 2019, 8, 3459. 


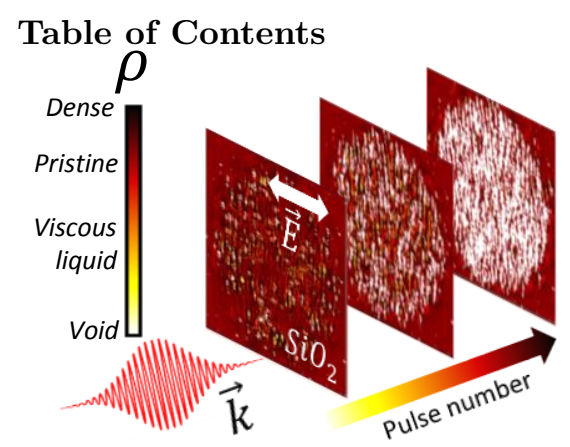

Nonlinear light-matter interaction driven by ultrashort laser pulses enables inscribing a variety of sub-wavelength void-like structures inside silica bulk, serving as optical elements with unique anisotropic and scattering properties. 3D modeling of the nanostructure genesis, evolution and self-organization to nanogratings is pioneered here by original coupled simulations considering both nonlinear propagation and material arrangement upon multipulse irradiation. 